\title{
EchoGéo
}

36 | 2016

Stratégies de villes et "modèles urbains"

\section{Les politiques de mobilité durable à Amman : vecteurs de promotion urbaine et outils de gouvernement contestés}

\section{Eliott Ducharme}

\section{(2) OpenEdition \\ Journals}

Electronic version

URL: https://journals.openedition.org/echogeo/14558

DOI: 10.4000/echogeo.14558

ISSN: 1963-1197

\section{Publisher}

Pôle de recherche pour l'organisation et la diffusion de l'information géographique (CNRS UMR 8586)

Electronic reference

Eliott Ducharme, "Les politiques de mobilité durable à Amman : vecteurs de promotion urbaine et outils de gouvernement contestés", EchoGéo [Online], 36 | 2016, Online since 30 June 2016,

connection on 11 August 2021. URL: http://journals.openedition.org/echogeo/14558 ; DOI: https:// doi.org/10.4000/echogeo.14558

This text was automatically generated on 11 August 2021.

EchoGéo est mis à disposition selon les termes de la licence Creative Commons Attribution - Pas d'Utilisation Commerciale - Pas de Modification 4.0 International (CC BY-NC-ND) 


\title{
Les politiques de mobilité durable à Amman : vecteurs de promotion urbaine et outils de gouvernement contestés
}

\author{
Eliott Ducharme
}

\section{Introduction}

1 L'évolution du rôle des villes dans le processus de mondialisation est devenue une thématique majeure de la recherche urbaine. Une série de travaux a approfondi la question de la montée en puissance des villes - et notamment des métropoles - dans un nouvel ordre mondial fondé sur la dématérialisation des échanges et la capacité de certains espaces à attirer les activités à forte valeur ajoutée (Sassen, 1991 ; Veltz, 1996). D'autres se sont intéressés aux modèles de gestion urbaine et ont insisté sur la place prépondérante des villes dans l'élaboration, la diffusion, la réception de modèles urbains dits néolibéraux dans un contexte de concurrence généralisée (Harvey, 1989, Brenner et Théodore, 2002). S'ajoutent des travaux sur les «policy transfers » ou "lesson-drawing» (Dolowitz et March, 2000), étudiant les modalités de diffusion et d'adaptation de modèles de gestion urbaine entre les institutions, notamment dans le cadre de relations Nord-Sud jugées asymétriques (Ward, 2000 ; Peck et Tickell, 2002).

2 La littérature récente tend néanmoins à rompre avec une analyse trop typologique et hiérarchisante des relations entre les villes. L'accent porté sur le caractère dynamique des interactions entre les territoires et des flux globalisés d'agents et de modèles remet en question l'essentialisme des dichotomies Nord/Sud et local/global (Mc Cann et Ward dir. 2011 ; Mc Cann, Roy et Ward, 2013). Des analyses plus fines des trajectoires urbaines des villes du Sud récusent le postulat de transferts unilatéraux en démontrant l'importance des arrangements locaux et insistent sur les relations de co-apprentissage entre les acteurs locaux et internationaux (Khirfan, 2011 ; Parnell et Robinson, 2012 ; 
Wood, 2014). Ainsi, l'approche en termes de villes globales, de "modernité » et de "développement» aurait conduit à éluder les dynamiques originales de la grande variété de villes «ordinaires» (Robinson, 2006) tandis que la critique de la ville néolibérale aurait conduit à minimiser la diversité des situations locales (Morange et Fol, 2014), réduisant le rôle des pays du Sud à celui de «suiveurs" (Harvey, 2005 cité par Morange et Fol, 2014).

3 Le cas de la Jordanie est dans ce contexte particulièrement intéressant à étudier du fait de l'ouverture du pays aux institutions internationales (FMI, OMC) après la crise économique de la fin des années 1980 et de l'importance des relations qu'il entretient avec les grands organismes humanitaires et de développement, suite à l'accueil d'un nombre important de réfugiés (Ababsa, 2011, 2013; Abu Sada et Challand,2012). Les nouveaux modèles de gestion publique qui en découlent - lesquels se traduisent notamment par un important recours aux privatisations et aux partenariats publicprivés - ont conduit de nombreux auteurs à aborder la production de la ville jordanienne par le prisme de la conversion au néolibéralisme (Parker, 2009; Daher, 2013 ; Debruyne et Parker, 2015), les conduisant à placer un focus critique sur les effets délétères d'un ordre urbain imposé par les grandes institutions néolibérales.

Cet article étudie les conditions de la mise en place à Amman de politiques de transport et de mobilité qui se réclament des principes du développement durable. Il entend replacer la problématique au niveau d'enjeux plus territoriaux liés d'une part aux ambiguïtés des processus de décentralisation et de démocratisation dans un régime autoritaire et clientéliste (Ababsa, 2015) et d'autre part aux revendications d'une classe moyenne éduquée qui trouve dans la problématique du cadre de vie un terrain de lutte. Cette réflexion s'appuie sur un travail de terrain qui mobilise des entretiens menés auprès de responsables locaux et nationaux de la mobilité en Jordanie ainsi que de représentants d'organismes de développement et de bailleurs de fonds. Il s'enrichit de la lecture critique des principaux documents de planification produits au niveau local et national et de la lecture quotidienne de la presse anglophone jordanienne et de tribunes publiées sur Internet. Nous verrons comment la mobilisation d'une expertise internationale, l'inscription dans des réseaux de villes et l'adoption de «bonnes pratiques » en matière de gestion durable de la mobilité urbaine s'inscrivent dans la volonté de rayonnement de la municipalité d'Amman tout en appuyant la revendication portée par une partie de ses élites d'une meilleure gestion de la vie urbaine. Nous nous interrogerons également sur les enjeux des recompositions territoriales et des conflits de pouvoir liés à l'adoption de ces modèles et à l'impératif de normalisation des pratiques et du paysage urbain dans une perspective de promotion de la capitale jordanienne.

5 En cela, nous questionnerons la capacité du paradigme du développement durable à légitimer la mise en place de politiques publiques contestées dans un contexte d'éclatement des structures de gouvernement urbain et interurbain. Cela nous conduira à discuter l'hypothèse proposée par certains auteurs proches des théories néomarxistes qui, reprenant les travaux de $\mathrm{M}$. Foucault sur la gouvernementalité, mettent en avant le pouvoir de consensus du discours de la durabilité au profit de politiques néolibérales hégémoniques (Reigner,2013; Swynguedouw, 2014). Ne remettant par ailleurs aucunement en cause le lien entre développement durable et capitalisme vert, nous verrons que les importants débats suscités par la volonté de mise en œuvre à Amman de politiques de mobilité durable ne se limitent pas à la simple 
question de l'adoption ou non de politiques néolibérales. Nous espérons en cela apporter un complément à la réflexion portée notamment par P.-A. Barthel et $\mathrm{E}$. Verdeil sur les conséquences de la mobilisation du développement urbain durable par les décideurs politiques dans les villes arabes, dans un contexte politique troublé (2013).

\section{Éléments de contexte : une capitale en forte croissance à la recherche de reconnaissance internationale}

6 Capitale macrocéphale regroupant près de la moitié de la population du pays, la municipalité d'Amman (Greater Amman Municipality ou GAM) a connu une croissance exponentielle suite à l'arrivée massive de réfugiés palestiniens, irakiens puis syriens depuis 1948. Dans les années 2000, suite à l'assassinat du Premier Ministre libanais Rafiq Hariri, la ville bénéficie d'un report des investissements notamment venus du Golfe, source d'importants flux de capitaux, occasionnant un boom de l'immobilier sans précédent (Ababsa, 2013).

Illustration 1 - L'expansion urbaine d'Amman (1946 - 1985)

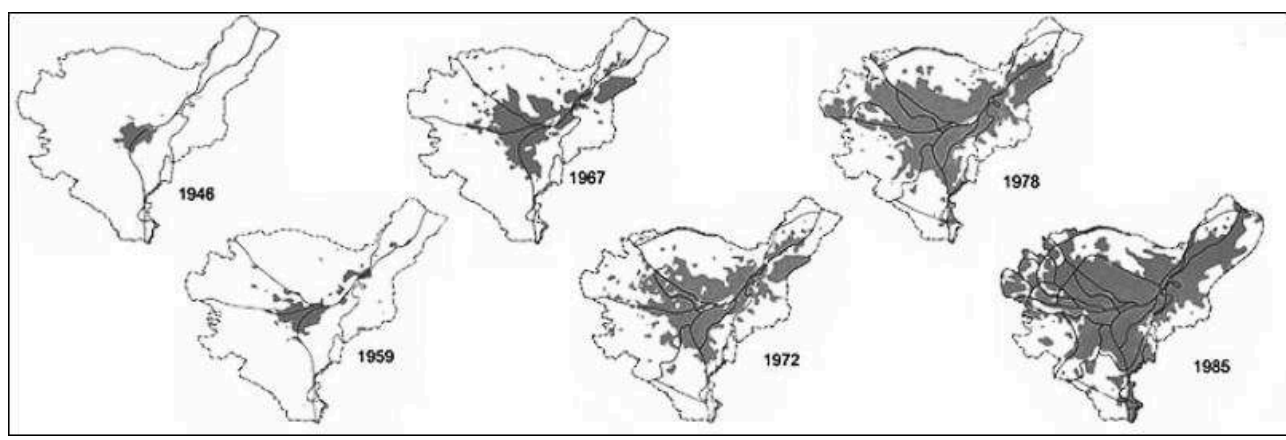

Source : Khirfan, 2011

7 Dans ce contexte, le GAM entreprend avec l'appui de partenaires internationaux une importante réflexion sur sa gestion urbaine, laquelle met fortement l'accent sur la durabilité. En 2004, la municipalité reçoit le soutien de la Banque Mondiale pour élaborer une "City-wide strategy" en partenariat avec l'Arab Urban Development Institute (AUDI) situé à Riyad, et l'Agence Française de Développement (AFD). Cette stratégie, renforcée notamment par le programme des Nations-Unies Cities Alliance, a pour but de promouvoir la capitale jordanienne comme cité pilote en termes de développement urbain durable à l'échelle de la région Moyen-Orient et Afrique du Nord (Verdeil, 2011). Elle est complétée en 2006 par la décision royale d'apporter au GAM un schéma directeur d'urbanisme censé planifier le développement urbain à horizon 2025 et hisser la capitale au rang des grandes métropoles internationales. Il s'agit notamment de créer un terrain propice au développement économique et à l'attraction des investissements étrangers dans une période de forte croissance, supposée pérenne (Beauregard et Marpillero-Colomina, 2010). Pour cela, le Roi nomme un nouveau maire, Omar Maani qui a pour consigne "d'inviter des experts du monde entier ", et de créer un exemple qui pourrait par la suite être répliqué dans d'autres villes jordaniennes (ibid). 
8 M. Maani sollicite ainsi trois cabinets d'architecture et d'urbanisme de Toronto dont certains membres effectueront des missions de plusieurs mois à Amman et il crée un think-tank au service de la municipalité l'Amman Institute (AI) dont la plupart des membres, largement surpayés par rapport au reste des employés municipaux, sont diplômés de grandes universités étrangères. Il est intéressant de noter la façon dont l'Amman Institute se décrit lors de sa candidature en 2010 à l'award for planning excellence décerné par le Canadian Institute of Planners - que les cabinets BearingPoint et PlanningAlliance avaient obtenu en 2008 pour le schéma d'urbanisme d'Amman :

«('Amman Institute) a créé un environnement institutionnel adapté aux jeunes et brillants esprits pour s'épanouir et apporter une contribution significative au développement de leur pays, retenant les talents et incitant ceux qui sont partis à revenir à la maison (...) Les employés de l'AI viennent de très célèbres institutions comme le MIT, UofT, London School of Economics, Arizona State University, University of Illinois et University of California at Berkley pour n'en citer que quelques-unes » (2010, p. 2, traduction de l'auteur).

9 .La cinquantaine de membres de l'Amman Institute et les urbanistes canadiens ont travaillé de concert au sein d'une structure nommée Program Management Unit (Khirfan, 2011), partageant ainsi leurs savoirs et visions de la ville dans des processus d'apprentissage à double sens (ibid). Le Master Plan qui en découle reçoit en 2007 le World Leadership Award in Town Planning, décerné par le World Leadership Forum (ibid).

Que retenir de cette entreprise de grande ampleur portée par la municipalité ? Amman, alors en forte croissance économique et démographique cherche à se placer sur le terrain des villes compétitives et tend à faire jouer un avantage comparatif important dans la région: une forte stabilité politique propice au développement économique, principalement tertiaire. La municipalité tend alors à promouvoir son image à l'international en valorisant une forme d'excellence urbanistique basée sur des savoirfaire transnationaux et la mobilisation des principes du développement urbain durable dont nous verrons les modalités ci-après.

\section{La mobilité durable comme stratégie de promotion urbaine à l'heure des villes en réseau}

\section{De la ville insoutenable à la mobilité durable}

11 Toute personne vivant ou ayant séjourné à Amman pourrait témoigner de la mauvaise qualité des conditions de transport. Longtemps soumis au laissez-faire, le secteur des transports publics, principalement constitué de micro-opérateurs ne possédant pour la plupart qu'un seul véhicule, peine à s'adapter à l'expansion urbaine croissante de la capitale. Les transports " municipaux » sont assurés par une compagnie de bus dont la privatisation dans les années 2000 s'est soldée par un échec, et qui est actuellement détenue à $61 \%$ par le GAM. La compagnie, largement sous-équipée, ne bénéficiant pas d'investissements suffisants, délivre un service de pauvre qualité qui suscite des plaintes récurrentes des usagers - au début de l'année 2015, une étudiante avait subi des brûlures suite à une déficience technique du matériel, suscitant d'importantes protestations (Jordan Times, avril 2015). Ainsi, les populations les plus démunies sont captives de transports publics peu performants et désorganisés tandis que les plus 
aisées se déplacent uniquement en voiture individuelle. Les conditions pour les piétonns sont très pénibles et les cyclistes inexistants.

Il va sans dire que l'image d'une ville de plus en plus congestionnée, polluée, parfois dangereuse au regard de la sécurité routière, et aux conditions de trajets difficiles s'accorde mal avec les objectifs de métropole compétitive, attractive, moderne, promue par les urbanistes de Toronto et de l'Amman Institute. En janvier 2014, le magazine d'affaires Venture publie un article intitulé « city of inconvenience » en écho à l'étude annuelle de l'Economist Intelligence Unit (EIU) sur la qualité de vie urbaine qui place Amman en $103^{\mathrm{e}}$ position dans un classement de 140 villes. Tout un paragraphe de l'article est consacré aux externalités négatives produites par les conditions déplorables de mobilité. En réponse, de grands projets d'amélioration des transports publics se mettent en place qui s'inscrivent dans un plan plus vaste de croissance verte pour la capitale. Ils mobilisent des réseaux d'expertise internationaux, des «bonnes pratiques » en termes de mobilité durable et font l'objet d'une importante promotion marketing.

En 2007, dans le cadre de l'élaboration du schéma directeur d'urbanisme d'Amman, la municipalité acquiert par décret royal la compétence en matière de gestion des transports publics urbains, auparavant détenue au niveau national. Un département de gestion du trafic et du transport public - dont la direction est confiée à un expert jordanien tout juste rentré des Etats-Unis où il avait fait sa thèse de doctorat et travaillé durant vingt ans - est alors créé avec le projet d'investir un milliard de dollars dans le transport sur les cinq prochaines années (Khirfan, 2011). Le plan de déplacement mis en place par la municipalité d'Amman vient conforter les objectifs de son schéma d'urbanisme: la volonté de rendre piétonnes certaines rues du centre ancien va de pair avec la valorisation du patrimoine bâti ; la planification de projets de transport à haute capacité, notamment de Bus Rapid Transit (BRT) s'intègre dans le développement de corridors de densification. Porteuse d'objectifs largement surévalués (tels que l'augmentation de la part modale des transports en commun de 13 à $40 \%$ entre 2010 et 2025), cette politique s'inscrit donc dans une séquence planificatrice de grande ampleur supposée donner à la capitale un rayonnement international.

\section{Le BRT, un outil prisé et stratégique en provenance des pays du Sud}

Le terme Bus Rapid Transit n'a pas réellement de traduction en français ${ }^{1}$. Nous utiliserons comme définition celle proposée par H.S. Levinson et al. dans une synthèse publiée dans un numéro du Journal of Public Transportation (2002) consacrée au BRT :

«Le BRT est un mode de transport roulant flexible qui associe des stations, des véhicules, des services, un site propre, et des technologies de transports intelligentes au sein d'un système intégré bénéficiant d'une image et d'une identité fortement positives. Les composantes du BRT sont conçues pour s'intégrer au marché qu'elles exploitent et à l'environnement physique dans lequel elles s'inscrivent, et peuvent être mises en place de manière incrémentale dans une pluralité de situations » (p. 2, traduction de l'auteur).

Fait intéressant à souligner, le BRT est un outil provenant « du Sud». Principalement développé en Amérique Latine - notamment avec les célèbres exemples de Curitiba au Brésil, où le premier BRT a été mis en place dans les années 1970 et de Bogota en Colombie, laquelle promeut son célèbre Transmilenio de par le monde - il commence également à connaître un important développement dans les pays asiatiques 
(Matsumoto, 2006). Prisé pour sa desserte de qualité à un coup largement inférieur à celui d'un tramway, pour ses aménagements urbains modernes et son système d'information en temps réel, le BRT fait office de panacée dans les pays en développement connaissant des problèmes croissants de mobilité. Il est ainsi la technologie de transport connaissant la plus forte progression au cours du XXI ${ }^{\mathrm{e}}$ siècle (Wood, 2015).

Illustration 2 - Le BRT de Curibita au Brésil

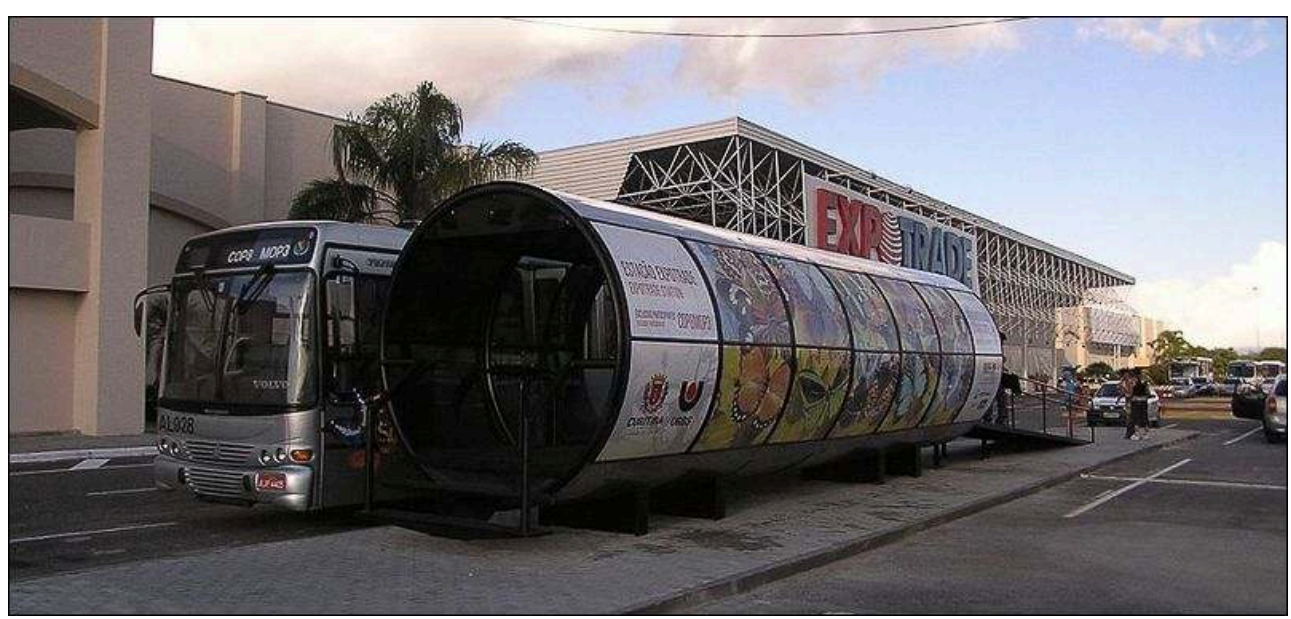

Les stations de bus sont également des outils de design urbain.

Source : Commons.wikimedia.

Largement promu par la Banque Mondiale notamment, le BRT est également un outil très prisé dans le cadre de projets de réduction des gaz à effet de serre qui associent les collectivités locales à des agences environnementales supranationales. C'est notamment le cas du Global Environnement Facility, mécanisme financier de UNFCCC qui crée en 2000 le programme intitulé «Promoting Environmentally Sustainable Transport ». Il se focalise principalement sur les solutions technologiques - bien qu'un outil complémentaire ait été créé en 2006 pour soutenir des mesures plus soft comme la planification (Bakker et Huizenga, 2010). Le recours au BRT est ainsi un bon moyen d'obtenir des fonds climats et des prêts internationaux. Ainsi, l'Agence Française de Développement - qui a par ailleurs apporté son soutien à la création d'un plan de déplacement urbain pour la capitale paru en 2010 - a accordé un prêt de 166 millions de dollars à la municipalité d'Amman pour la réalisation d'une première phase de BRT à partir de cette même année. 
Illustration 3 - Répartition des financements du GEF liés au transport

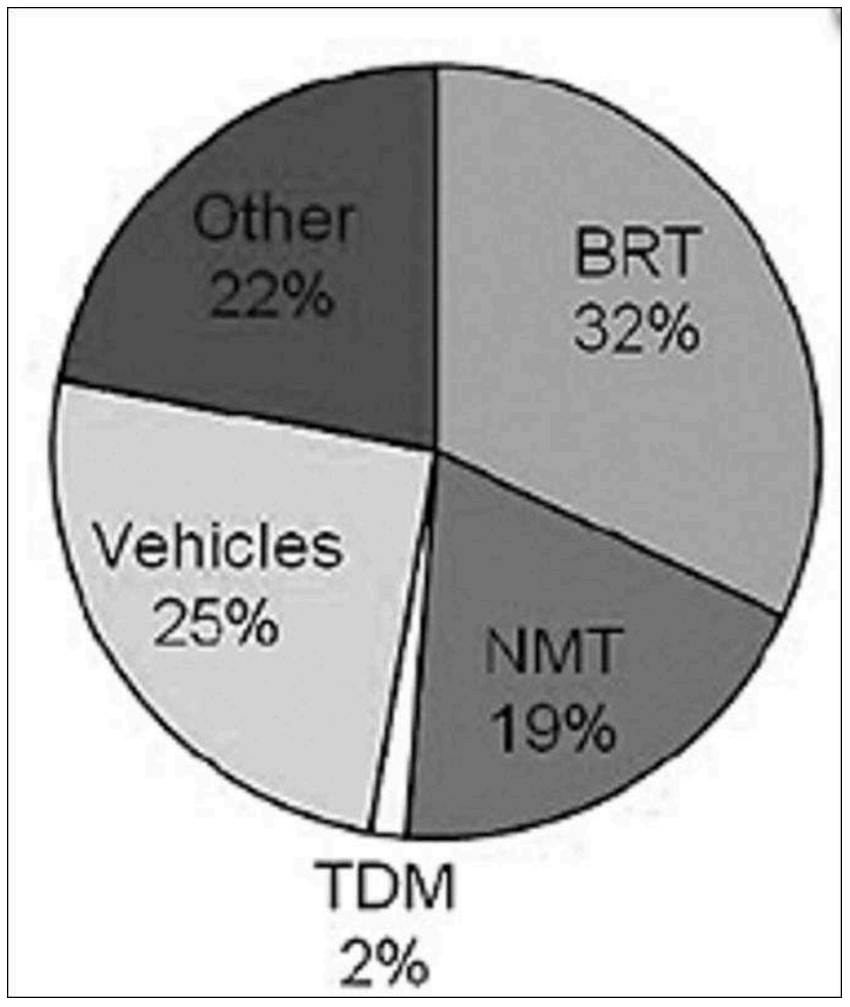

Source : Bakker et Huizenga, 2010

17 Amman, qui souhaite adopter un positionnement fort en termes de durabilité urbaine entend largement mobiliser l'outil BRT dans le cadre de son plan de croissance verte, l'Amman Green Growth Program, élaboré par l'Amman Institute en partenariat notamment avec l'unité de la finance carbone de la Banque Mondiale. Ce plan traite de domaines variés de la gestion municipale - dont l'eau, l'énergie, la gestion des déchets, la nature en ville avec la réduction des ilots de chaleurs et les transports - et propose des mesures pour réduire les émissions de gaz à effet de serre, améliorer la qualité de l'air etc. Il s'inscrit dans la démarche du schéma directeur d'Amman et poursuit les mêmes objectifs de renouvellement de la gestion urbaine et de mise en visibilité de la capitale. La mise en place de BRT doit ainsi conduire à une réduction de 6,6 milles tonnes de $\mathrm{CO}^{2}$ par an, et une réduction totale 280 tonnes d'ici les 30 prochaines années (the city fix, avril 2013).

Cette stratégie de croissance verte porte également ses fruits en termes de communication internationale : un article publié en 2012 par U.S. Green Building Council (usgbc.org, janvier 2012), encourage les villes américaines à prendre exemple sur Amman ; le New-York Times titre en 2010 « Sidewalks and an identity sprout in Jordan's capital». Elle permet également à Amman de s'insérer dans des réseaux de villes prestigieux, tels que le $C 40$, réseau de métropoles issu d'une démarche entreprise en 2005 par le maire de Londres et relayée par ses homologues de Toronto et de New-York et dont le but est de "s'emparer de la question du changement climatique en développant des politiques et programmes générant des réductions mesurables du gaz à effet de serre et du risque climatique 》(C40.org). Amman fait partie de son comité de pilotage ainsi que du réseau " 100 Resilient Cities" parrainé par le Rockfeller Institute. Ce qui lui permet ainsi de s'insérer dans les réseaux de la résilience, notion à la mode qui s'opérationnalise et 
fédère, non sans récupérations dangereuses (voir notamment Djament-Tran et Reghezza-Zitt, dir, 2012).

19 Ainsi, à travers l'étude du développement de politiques de mobilité urbaine durables à Amman, nous avons essayé de montrer que les réseaux d'acteurs mobilisés dans le cadre de ce changement de modèle de gestion urbaine sont multiples. Loin de se réduire à une lubie des bailleurs de fonds, la mobilisation du paradigme du développement durable par la ville d'Amman se fait dans le cadre d'une stratégie de positionnement à l'échelle régionale voire internationale dans un contexte de concurrence entre les villes. La présence de techniciens surdiplômés, la maîtrise des ressorts de la mobilisation de l'aide internationale permettent au GAM d'avoir un réel contrôle sur ce processus, contrairement aux affirmations quelque peu tranchées des géographes radicaux notamment qui réduisent le rôle des pays du Sud à celui de « suiveurs » (Harvey, 2005 cité par Morange et Fol, 2014).

De plus, loin de se résumer à une simple conversion au néolibéralisme par le biais du capitalisme vert et à une volonté de promotion urbaine par le développement d'aménités environnementales et la minimisation des nuisances urbaines, l'adoption de mesures pour la mobilité durable mobilise un écosystème d'acteurs plus vaste que les simples décideurs politiques et techniciens municipaux. Elle questionne plus profondément les modes de gestion urbaine, la question des échelles territoriales d'action, et réveille des tensions sociales existant dans la société jordanienne.

\section{Ici et ailleurs. Imaginaires transnationaux, recompositions territoriales et tensions sociales}

\section{Les réseaux locaux de la ville durable}

21 Il est intéressant d'étudier plus en détail l'emprise locale de ces « réseaux de la mobilité durable » et les acteurs qui défendent ce principe sur différentes scènes politiques. En effet cela permet de conforter l'argument premier selon lequel les institutions et les sociétés du Sud ne sont pas de simples réceptacles pour des principes de gestion venus du Nord et de montrer que l'adoption de certaines positions peut être motivée par une grande diversité de raisons selon les personnes. D'autre part, l'analyse des liens entre développement durable et rationalité néolibérale, mis en avant par plusieurs chercheurs (Béal, 2011 ; Reigner, 2013 ; Swynguedouw, 2014), amène à considérer le recours à la mobilité urbaine durable comme un outil de gouvernement qui permet de rassembler une groupe d'acteurs disparate autour d'un objectf de normalisation des pratiques urbaines supposée améliorer l'image de la capitale tout en justifiant le renforcement des pouvoirs municipaux.

La Jordanie possède un terreau associatif fertile du fait de sa proximité avec les bailleurs occidentaux qui valorisent les projets «portés par la société civile. Elle manifeste la volonté de se donner une image de monarchie constitutionnelle libérale et il existe dans le pays une jeunesse éduquée et dynamique. Aussi de nombreuses organisations portent des revendications notamment en termes de protection de l'environnement, défense des droits de l'Homme, reconnaissance du droit des femmes, promotion d'initiatives citoyennes etc. Certaines d'entre elles bénéficient ainsi de soutiens financiers et techniques des bailleurs internationaux. C'est le cas notamment de l'International Youth Foundation qui, avec le soutien de l'USAID produit en 2014 un 
rapport sur les conditions difficiles de mobilité des jordaniens - et notamment des jordaniennes - et ses conséquences dans l'accès à l'emploi. Plusieurs associations à l'ancrage plus local exercent une activité de lobbying importante auprès du gouvernement pour le développement des transports publics.

C'est notamment le cas de la campagne Ma'an Nasel (qui se traduit par « ensemble nous arrivons ») lancée au début de l'année 2015 dans le but d'obtenir des autorités la reconnaissance du transport public comme une priorité nationale. Cette campagne est portée par la plateforme citoyenne jordanienne Taqaddam qui se présente comme une plateforme activiste interactive et informative, active sur le web mais également impliquée dans l'organisation de débats, de campagnes de sensibilisation et d'information autour de la promotion d'une « citoyenneté moderne et démocratique ». Ses combats portent aussi autour du respect des libertés individuelles, de la justice sociale et de la durabilité environnementale. Elle est financée par des philanthropes jordaniens et refuse par ailleurs toute forme de financement étranger. Forte de quelques soutiens au Parlement, l'association mène une série d'actions visant à susciter la prise de conscience des enjeux de l'amélioration du transport public. Parmi ces actions, la publication d'une étude réalisée par une autre association, le « Center for the Study on the Built Environement » (CSBE) en Juin 2015 sur les conditions difficiles des usagers du transport public à Amman, qui a été l'occasion d'un événement médiatisé rassemblant les principaux décideurs dans le domaine du transport dont la ministre des transports.

Les membres de ces deux dernières associations appartiennent à ce que l'on pourrait nommer une élite internationale jordanienne. Très éduqués, maîtrisant parfaitement l'anglais et ayant pour beaucoup fait leurs études (longues) à l'étranger, ils sont très au fait $d u$ fonctionnement urbain des villes occidentales auquel ils se réfèrent et qu'ils présentent parfois de manière quelque peu idéalisée, comme en témoigne le montage ci-dessous publié sur la page facebook du CSBE.

Illustration 4 - Page facebook du CSBE

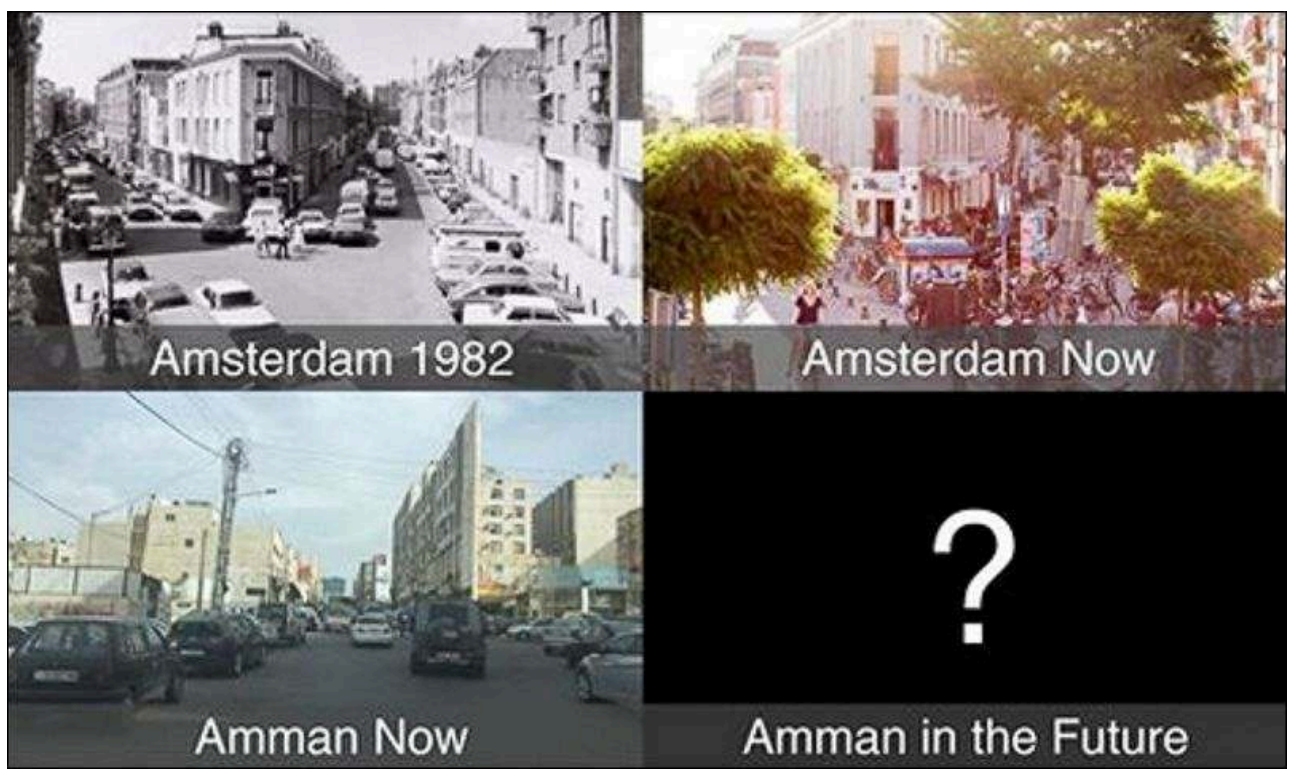

Source : CSBE, page facebook. 
personnes ont de bonnes relations avec les décideurs du GAM qu'ils connaissent pour certains personnellement (un des membres fondateurs de Ma'an Nasel est un ancien employé de la municipalité d'Amman). Ils forment ce que l'on pourrait appeler une "communauté épistémique ", "un ensemble d'acteurs qui partagent un ensemble de valeurs, des diagnostics et des solutions afin de traiter des problèmes en vue d'améliorer le bienêtre humain" (Bossy et Evrard, 2010). Cette communauté va former ce qu'A. Allal a nommé une "configuration développementiste internationale », dans son étude de l'apprentissage des politiques de démocratisation et de "bonne gouvernance " au Maroc en Tunisie: un "système d'acteurs, composé d'élites qui sont à la fois cosmopolites, marquées par un ensemble de dispositions spécifiques, par une culture professionnelle commune, et plus ou moins ancrées dans la société nationale » (Allal, 2010).

Notons que ces acteurs, qui se réclament de la justice sociale, vont faire cause commune avec la municipalité dans la mise en place de ces mesures de mobilité durable notamment durant la construction controversée de la première phase du BRT dont nous reparlerons - et ce malgré les incertitudes en terme d'équité et de justice sociospatiale liées à ce projet. En effet, dans le cadre de projets controversés de renouvellement urbain et sans entrer dans les détails, la « rationalisation » du système de transport a notamment occasionné le déplacement en périphérie des deux stations de bus les plus importantes d'Amman, garantes de l'accès au centre de populations modestes issues des villes périphériques. De plus les choix opérés notamment dans le tracé du BRT laissent dubitatif quant à l'objectif réel de cet outil, entre l'amélioration de la mobilité pour les plus démunis ou la création d'un outil de marketing urbain. La mobilisation du paradigme de la durabilité, de «bonnes pratiques » internationales et d'un imaginaire fantasmé de la ville occidentale semble servir outil de gouvernementalité au profit d'une certaine vision de l'ordre urbain. Ces modèles sont en effet producteurs d'une forme de consensus qui légitime l'action de décideurs principalement préoccupés par la promotion de la ville sur la scène internationale et la normalisation des comportements urbains. Nous verrons cependant que ce consensus peine à s'étendre au-delà de la " communauté épistémique » précédemment décrite et que le pouvoir normalisateur de la mobilisation du paradigme du développement durable se heurte à d'importants conflits territoriaux et à des luttes de pouvoir vivaces.

\section{Stratégie urbaine internationale et conflits locaux. Jeux d'échelles et jeux de pouvoir}

La mobilisation de slogans internationaux tels que «développement durable » et «bonne gouvernance », appliqués à la gestion urbaine est également une opportunité pour la municipalité d'Amman. En effet cela lui permet de gagner en autonomie dans un contexte de fortes tensions entre le caractère très bureaucratique et centralisé de l'État jordanien et sa volonté de créer des entités d'exception gérées selon des principes néolibéraux et supposées jouer un rôle moteur dans l'économie du pays (Debruyne et Parker, 2015). La capitale, macrocéphale, possède ainsi un budget per capita largement supérieur aux autres municipalités. De plus, le GAM bénéficie depuis 1995 d'un statut territorial d'exception : son maire est nommé par le gouvernement parmi les proches du Roi de même qu'un tiers de son conseil municipal. La capitale bénéficie également de ressources humaines incomparables eu égard au personnel municipal jordanien, habituellement pléthorique et peu formé. Dans le cadre du processus national 
controversé de fusion des municipalités engagé depuis 2001, Amman englobe en 2007 plusieurs communes limitrophes en son sein, doublant ainsi son périmètre d'action et s'assurant notamment la maittrise du front futur d'urbanisation vers le Sud-Ouest (AlHaija, Potter, 2013).

Illustration 5 - L'expansion municipale du GAM en 2007

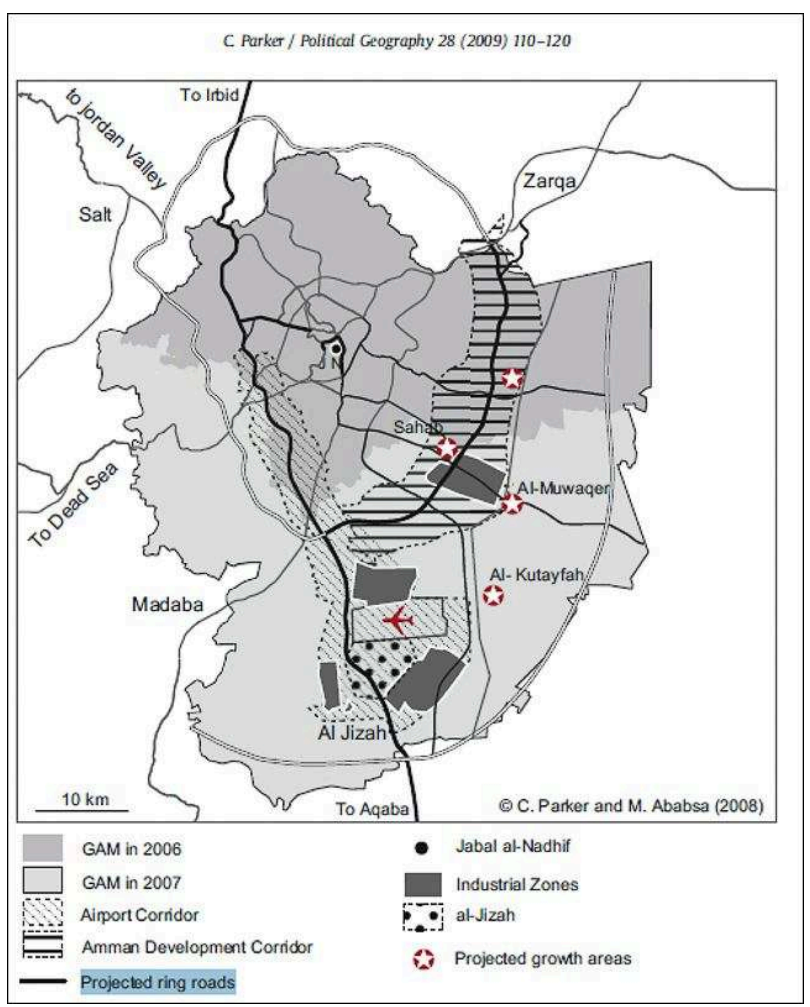

Source : Parker, 2009

Le recours à l'aide étrangère consolide également la position de la municipalité qui mobilise la rhétorique de la «bonne gestion ». Dans le cadre de la mise en place de la première phase du BRT, celle-ci a bénéficié d'un prêt non-souverain de la part de l'AFD - fait impensable dans d'autres municipalités - lequel ne se délivre pas sans conditions. Ainsi, l'État jordanien a dû s'engager notamment à rembourser au GAM le manque à gagner occasionné par la suspension des contraventions routières (représentant plus de 20 millions de dinars) - alors supposée apaiser la population dans le contexte des Printemps Arabes - ainsi qu'à ne plus intervenir dans les affaires de la municipalité. C'est également sous couvert de cette «bonne gestion » que le GAM défend son statut d'exception, et notamment la gestion des transports publics, face au ministère des Transports qui dénonce l'absurdité de cette différence de statut, source de conflits dans la mise en place de projets intercommunaux. La mise en avant d'une planification intégrée de l'urbanisme et des transports, conforme aux bonnes pratiques internationales, sert d'argument à la municipalité pour conserver ses prérogatives.

Cependant, dans la tourmente des Printemps Arabes, les acquis de la municipalité se voient fortement contestés. Les protestations pour la démocratie et contre la corruption vont s'accompagner d'une remise en cause des nouvelles frontières municipales, notamment par les leaders tribaux qui s'étaient sentis lésés au moment de leur découpage (Absbsa, 2013). Le projet de BRT - par ailleurs très contesté - est 
également suspendu pour cause d'irrégularité dans les appels d'offres, le maire d'Amman Omar Maani est alors destitué et l'Amman Institute dissous. Dans un communiqué au Jordan Times, le directeur du département des transports de la municipalité dénonce une affaire politique (mai 2012). Selon un cadre du GAM, la montée en puissance de la municipalité a inquiété certains membres du Parlement habitués à bénéficier de passe-droits dans la gestion des affaires municipales. La technicisation du gouvernement de la municipalité, l'affirmation des logiques d'efficience, peuvent être perçues comme un danger pour les anciennes élites. De plus, le BRT, dont les travaux ont été lancés dans la hâte sans réelle consultation a été mal perçu par une partie des habitants, notamment du fait de l'importante gêne occasionnée pour la circulation automobile (Pinel, 2013). De fait, une partie des questions liées au BRT ont été laissées sans réponse de la part des autorités : quid notamment du devenir des très nombreux micro-opérateurs qui assurent la majeure partie de la desserte en transport public? Quelle implication des usagers et habitants dans la définition et le suivi du projet alors que tous les documents liés au BRT ont été rédigés en anglais?

Illustration 6 - Les stigmates de l'interruption du BRT en 2011 : deux kilomètres de voie à l'abandon

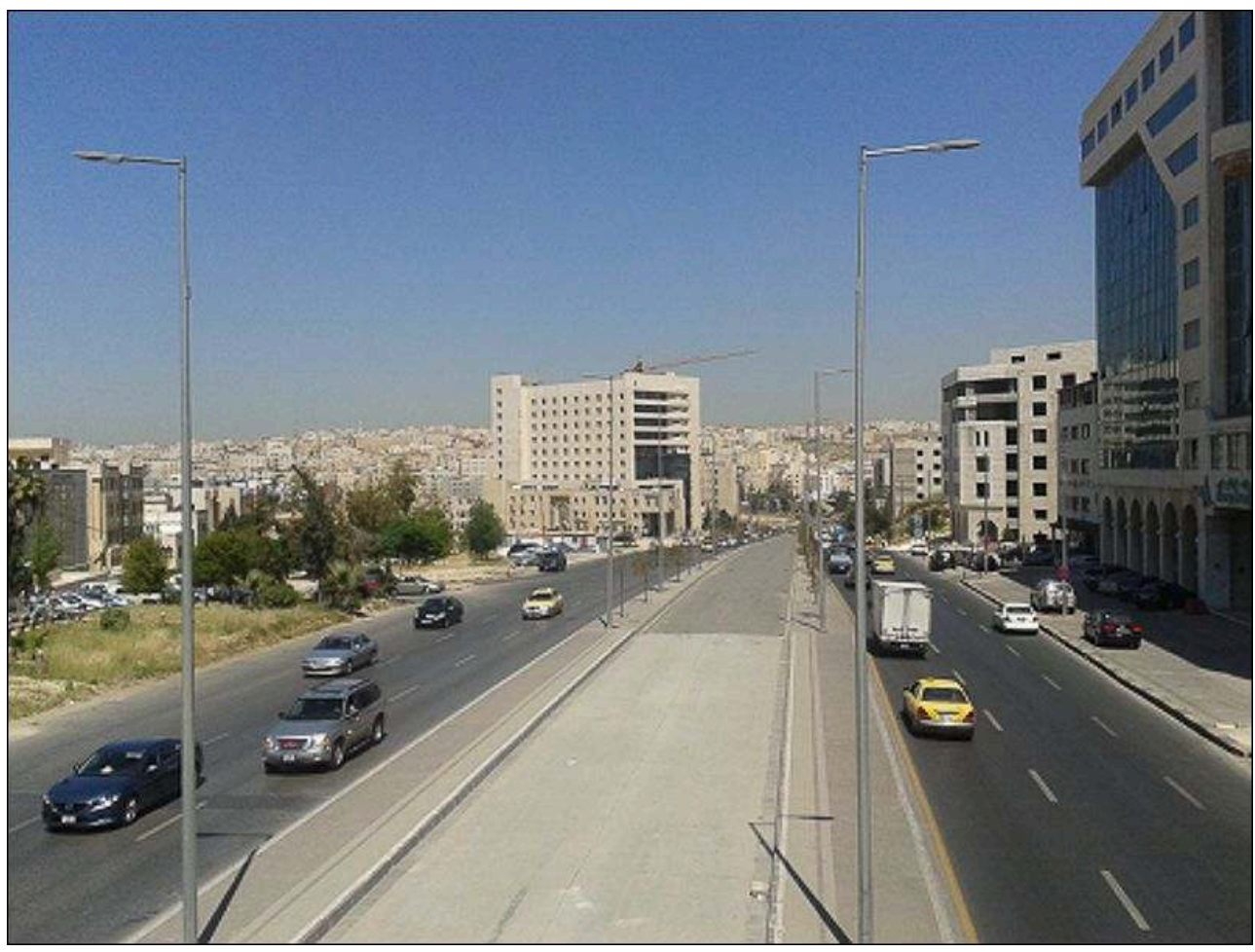

Auteur : Lorenza Tomasoni.

Alors que les travaux du BRT ont finalement repris au printemps 2015 et que sa cote de popularité semble, d'après les récents sondages, avoir augmenté, quelles conclusions pouvons-nous tirer des fortes crispations liées à ces politiques de mobilité durable? Nous aurions également pu évoquer les projets controversés de piétonisation, l'éviction du grand marché aux vêtements, prisé des classes populaires, pour cause de projet urbain supposé valoriser les circulations douces et les "solutions durables » etc. - Le « changement de paradigme »- selon les termes du maire d'Amman (Venture, 2014) que la municipalité entend impulser par la dynamique du développement durable est 
porté par une multitude d'acteurs nationaux ou internationaux aux rationalités multiples, parfois divergentes sur le fond. Se pose notamment la question de l'opportunité ou non du mode de gestion néolibéral. Divers acteurs internationaux jouent un rôle ambivalent, non dénué d'arrières pensées géostratégiques et d'intérêts commerciaux qui trouvent dans le développement durable un puissant outil de légitimation ${ }^{2}$. Par ailleurs, des techniciens municipaux désireux de promouvoir une capitale compétitive, en accord avec les standards internationaux, assoient leur légitimité par le recours aux «bonnes pratiques internationales» de gouvernement. Enfin, des élites urbaines transnationales sensibles aux questions d'équité souhaitent une ville mieux gérée, un gouvernement plus démocratique et conforme aux modèles occidentaux.

31 Cependant, cet idéal urbain, à bien des égards utopique, nourri d'imaginaires fantasmés de la ville moderne et verte, rencontre de nombreux obstacles. Tout d'abord les pratiques quotidiennes des habitants qui sont peu enclins à renoncer à la voiture individuelle. Ensuite les 3500 micro-opérateurs de transport public dont c'est le gagnepain et pour lesquels la question de leur devenir n'a pas été clairement abordée. Enfin, il existe des mécanismes politiques obscurs et de nombreuses ambiguïtés liées à des processus de décentralisation et de démocratisation hésitants et inachevés (Ababsa, 2015).

\section{Conclusion}

Dans cet article, nous avons tenté de nuancer les thèses de la conversion au néolibéralisme, de l'unilatéralité des transferts des "bonnes pratiques » et de la passivité des pays du Sud dans l'adoption des modèles internationaux. De toute évidence la mobilisation de la thématique de la durabilité urbaine dans le cadre de l'élaboration d'un schéma directeur d'urbanisme modèle est le fruit d'une stratégie urbaine maîtrisée. La municipalité d'Amman a su mobiliser des réseaux internationaux et tirer parti de leurs apports dans un contexte où elle avait là une opportunité de se démarquer des autres villes de la région. Nous avons vu cependant que ce " changement de paradigme » de la gestion urbaine ne se réduisait pas à une volonté de marketing urbain et de promotion internationale. Il s'agit également d'un positionnement dans le système de gouvernance national qui fait valoir une légitimité par l'expertise et par la mobilisation de "bonnes pratiques» de gestion urbaine. Cependant, cette vision utopique, et à plusieurs égards hors-sol de capitale moderne doit faire face à d'importantes contradictions. Les cinq dernières années ont donc été une période d'ajustements constants entre la volonté de planification d'une urbanité conforme aux bonnes pratiques internationales et la réalité du terrain, entre un idéal de bonne gestion et les pratiques sociales et spatiales de la population. Outil de promotion urbaine et de mise en réseau de la capitale d'une part, moyen de captation de financements internationaux dans le cadre de projets verts d'autre part, la mobilisation $\mathrm{du}$ développement urbain durable est également un moyen d'autonomisation de la capitale et de normalisation des pratiques urbaines des populations qui ne sont pas dupes à ce sujet. Il $\mathrm{y}$ a donc plusieurs niveaux d'enjeux pour la construction de ce nouveau modèle de gestion urbaine. Mais aussi plusieurs grilles de lecture dans la critique de ce modèle, que ce soit en termes de politiques néocoloniales, 
d'injustices spatiales, de pouvoir ou de domination. Toutes ayant leur logique et leur intérêt dans la perspective d'une recherche de modèles urbains plus justes.

\section{BIBLIOGRAPHY}

Ababsa M., 2011. Introduction. Citoyenneté et question urbaine en Jordanie. In Ababsa M. et Daher R. dir, Villes, pratiques urbaines et construction nationale en Jordanie, Cahiers de l'Ifpo, $\mathrm{n}^{\circ}$ 6, Beyrouth, Presses de l'Ifpo, p. 15-37.

Ababsa M. dir., 2013. Atlas of Jordan. History, Territories and Society. Beyrouth, Presses de l'Ifpo, $485 \mathrm{p}$.

Ababsa M., 2015. Jordanie : la décentralisation par décision centralisée et la démocratie par volonté royale. In Harb M. et Atallah, S. dir., Local governments and public goods: assessing decentralization in the Arab world, Beirut, LCPS, p. 139-188.

Abu Sada C., Chaland B., dir, 2012. Le développement, une affaire d'ONG ? Paris, Karthala, 240 p.

Al Haija A., Potter R. B., 2013. Greater Amman: metropolitan growth and scenarios for sustainable urban development. In Gambardella C., Heritage, Architecture, Landesign: focus on Conservation, Regeneration, Innovation, Le vie dei Mercanti: XI Forum Internationale di Studi, La Scuola di Pitagora Editrice, Napoli, Italy.

Allal A., 2010. Les configurations développementistes internationales au Maroc et en Tunisie : des policy tranfers à portée limitée. Critique international, 3/2010 ( $\left.n^{\circ} 48\right)$, p. 97-116.

Amman Institute, 2010. CIP Award 2010, summary submission. Amman, 137 p.

Bakker S., Huizenga C., 2010. Making climate instruments work for sustainable transport in developing countries. Natural Resources Forum, 34(4), p. 314-326.

Barthel P.A., Verdeil, E., dir., 2013. Ville arabes, villes durables ? Enjeux, circulations et mise à l'épreuve de nouvelles politiques. Environnement Urbain/Urban Environment, vol. 7.

Beal V., 2011. Ville durable et justice sociale. Ce que le développement durable nous dit de la production de l'urbain. In Beal V., Gautier M., Pinson G., Le developpement durable changera-t-il la ville? Le regard des sciences sociales, Publications de l'Université de Saint-Etienne, p. 239-259.

Beauregard R., Marpillero-Colomina A., 2011. More Than a Master Plan: Amman 2025. Cities, 28, p. 62-69.

Bossy T., Evrard A., 2010. Communauté épistémique. In Boussaguet L. et al., Dictionnaire des politiques publiques, Presses de Sciences Po, « Références », p. 140-147.

Brenner N., Theodore N., 2002. Spaces of Neoliberalism: Urban Restructuring in Western Europe and North America. Blackwell, Oxford.

Comstock M., 12 janvier 2012. City-wide Clean Development Mechanism: A Framework for Empowering Cities. http://usgbc.org 
Daher R., 2013. Neoliberal Urban Transformations in the Arab City Meta-Narratives, Urban Disparities and the Emergence of Consumerist Utopias and Geographies of Inequalities in Amman. Urban environment, Volume 7, p. 99-115.

Debruyne P., Parker C., 2015. Reassembling The Political: Placing Contentious Politics In Jordan. In Gerges F. A., dir, Contentious politics in the Middle East: popular resistance and marginalized activism beyond the Arab uprisings, p. 437-467.

Djament-Tran G., Reghezza-Zitt, M., dir., 2012. Résiliences urbaines : les villes face aux catastrophes. Paris, Le Manuscrit, 364 p.

Freij M., 2015. Hashemite University students to boycott AutoBus for one week over "poor services". Jordan Times, 19/04/15.

Dolowitz D. P., Marsh D., 2000. Learning from Abroad: The Role of Policy Transfer in Contemporary Policy-Making. Governance, 13, p. 5-23.

Harvey D., 1989. From managerialism to entrepreneurialism: the transformation in urban governance in late capitalism. Geografiska Annaler, Vol. 71b, n 1, p. 3-17.

Khirfan L., 2011. From Toronto to Amman: The Cross-National Transfer of Planning. Planning Theory \& Practice, 12, 4, p. 525-547.

Levinson H.S. et al., 2002. Bus Rapid Transit: An Overview. Journal of Public Transportation, 5, 189 p.

Matsumoto N., 2006. Analysis of policy processes to introduce Bus Rapid Transit systems in Asian cities from the perspective of lesson-drawing: cases of Jakarta, Seoul, and Beijing. Better Air Quality Workshop, Yogyakarta, Indonesia, $20 \mathrm{p}$.

McCann E., Ward K., dir., 2011. Mobile urbanism: Cities \& policy-making in the global age. Minneapolis, University of Minnesota Press, 256 p.

McCann E., Roy A., Ward K., 2013. Assembling/Worlding cities. Urban Geography, p. 581-589.

Morange M., Fol S., 2014. Ville, néolibéralisation et justice. Justice spatiale / spatial justice, $\mathrm{n}^{\circ} 6$, juin 2014.

Neimat K., 2012. Bus Rapid Transit still best option for Amman, but project 'politicised' - experts. Jordan Times, 8 mars 2012.

Parker C., 2009. Tunnel-bypasses and minarets of capitalism: Amman as neoliberal assemblage. Political Geography, vol. 28, p. 110-120.

Parnell S., Robinson J., 2012. (Re)theorizing Cities from the Global South: Looking Beyond Neoliberalism. Urban Geography, 33, 4, p. 593-617.

Peck J., Tickell A., 2002. Neoliberalizing Space. Antipode, 34, p. 380-404.

Pinel A., 2013. Lorsque le Sud innove en matière durable. Le projet de transport en commun d'Amman. In Barthel P.A., Verdeil E., dir., Ville arabes, villes durables ? Enjeux, circulations et mise à l'épreuve de nouvelles politiques, Environnement Urbain/Urban Environment, vol. 7.

Reigner H., 2013. Vers une gouvernance neohygieniste des conduites? Les formes renouvelées de la domination dans la ville pluraliste. Working papers du Programme 'Cities are back in town', 45 p. Robinson J, 2006. Ordinary cities: between modernity and development. London, Routledge, 224 p. Sassen S., 1991. The Global City: New York, London, Tokyo. Princeton, Princeton University Press, $480 \mathrm{p}$. 
Slackman M., 2010. Sidewalks, and an Identity, Sprout in Jordan's Capital. The New-York Times, 23 février 2010.

Swyngedouw E., 2014. The Violence of Sustainable Urbanity. Metropolitics.

Veltz P., 1996. Mondialisation, villes et territoires : Une économie d'archipel. Paris, PUF, coll. « Quadrige », 288 p.

Venture, rédaction, 2014. City of Inconvenience. Venture, janvier 2014.

Venture, rédaction, 2014. Biltaji’s Plan For Amman. Venture, janvier 2014.

Verdeil É. 2011. Villes, énergie et développement durable en Jordanie : entre néolibéralisme et improbable décentralisation. In Barthel P.A., Zaki L., dir., Expérimenter la « ville durable » au sud de la Méditerranée. Chercheurs et professionnels en dialogues, Editions de l’Aube, p. 291-319.

Ward S., 2000. Transnational planners in a postcolonial world. In Healey P., Upton R., dir, Crossing Borders: International Exchange and Planning Practices, London, Routledge, p. 47-72.

Wood A., 2014. Moving policy: global and local characters circulating bus rapid transit through South African cities. Urban Geography, 35:8, p. 1238-1254.

Zevitz E., 2013. Will Jordan gets its advanced bus system? The city fix, 17 avril 2013.

\section{NOTES}

1. Bien que l'on soit tenté de comparer le BRT aux Bus à Haut Niveau de Service (BHNS) mis en place dans les villes européennes, les caractéristiques morphologiques et socio-économiques de ces dernières rendent la comparaison difficile (voir notamment les nombreuses publications du CEREMA à ce sujet).

2. En cela l'Agence Française de Développement joue un rôle ambigu, notamment du fait de sa participation en juin 2015 à au forum urban smart. Organisé par le service économique de l'ambassade de France en Jordanie il rassemblait des industriel français et des décideurs et techniciens jordaniens autour des questions de durabilité urbaine au sein desquelles le transport avait une place prépondérante.

\section{ABSTRACTS}

This paper studies the context of the implementation of sustainable development oriented urban

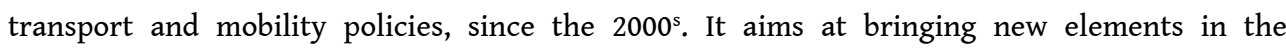
discussion on how globalization and neoliberalization processes affect cities of the global South. It insists on Amman municipality's active role and strategy in mobilizing international "best practices" and actor networks. The paper also underlines the plurality of the territorial scales involved in these policy transfers, between the city branding issues at the regional, even international level and the affirmation of the municipality's territory and competencies at the national level. Finally, it describes the important contestations that are shooking the implementation of these policies, which became a field for power-based territorial conflicts. 
Cet article étudie les conditions de la mise en place à Amman de politiques de transport et de mobilité se réclamant des principes du développement durable depuis la deuxième moitié des années 2000. Il entend apporter des éléments de réponse au débat portant sur la manière dont les villes dites du Sud sont affectées par les phénomènes de mondialisation et de néolibéralisation en mettant en lumière le rôle actif et stratégique de la municipalité d'Amman dans la mobilisation de modèles issus de « bonnes pratiques » internationales de gestion urbaine et des réseaux qui les promeuvent. Il insiste également sur la pluralité des échelles impliquées dans ces transferts de modèles, entre les enjeux de promotion urbaine à l'échelle régionale voire internationale et d'affirmation du territoire et des compétences municipales au niveau national. Il fait enfin état des fortes contestations qui ont ébranlé la mise en place de ces politiques devenues le terrain de luttes de pouvoirs territoriales.

\section{INDEX}

Mots-clés: transfert de politique publique, Jordanie, néolibéralisme, réseau d'acteurs, politique de transport

Keywords: policy transfers, jordan, neoliberalism, actors networks, transport policies

Subjects: Sur le Champ - Sur le Terrain

\section{AUTHOR}

\section{ELIOTT DUCHARME}

Eliott Ducharme, eliott.ducharme@laposte.net, est urbaniste. Il a publié récemment :

- Ducharme E., 2015. La gestion de l'eau en Jordanie face à la « crise syrienne » : entre transition néolibérale et tensions sociales. Métropolitiques, 24 juin 2015. 